\title{
MRI of Acute Abdominal and Pelvic Pain in Pregnant Patients
}

\author{
Katherine R. Birchard ${ }^{1}$ \\ Michele A. Brown ${ }^{1,2}$ \\ W. Brian Hyslop ${ }^{1}$ \\ Zeynep Firat ${ }^{1}$ \\ Richard C. Semelka ${ }^{1}$
}

OBJECTIVE. The purpose of this study was to show the usefulness of MRI in the evaluation of pregnant women with acute abdominal or pelvic pain.

SUBJECTS AND METHODS. All MRI studies of pregnant patients who were referred for examination because of acute abdominal or pelvic pain between June 2002 and May 2004 were included in this study $(n=29)$. The use of MRI was at the discretion of the clinician. Fetal sonography was performed in all patients before any other imaging. A complete abdominal sonographic examination was performed in six patients before MRI. In the remaining 23 patients, MRI was the choice for primary imaging. Multiplanar multisequence MR images of the abdomen and pelvis were obtained in each patient. Unenhanced images were reviewed by an experienced radiologist to determine whether a diagnosis could be made without the administration of gadolinium. In 22 of 29 studies, gadolinium was not administered. The prospective clinical MR interpretations were compared with follow-up medical, surgical, and obstetric records to determine the correctness of the interpretation. All patients were followed up until the date of article submission or until the date of final chart entry.

RESULTS. Correlation of prospective clinical MR interpretations with follow-up medical records showed correct identification of disease entities in all but one patient. In one patient, torsion of the ovary was neither described prospectively nor seen in retrospect. The following disease processes were correctly identified using MRI: appendiceal abscess $(n=1)$, appendicitis $(n=2)$, intraabdominal and rectus muscle abscess $(n=1)$, intussusception $(n=1)$, pancreatitis $(n=1)$, ulcerative colitis $(n=1)$, Crohn's disease with diffuse peritoneal inflammation $(n=1)$, bilateral adrenal hemorrhage $(n=1)$, pyelonephritis $(n=2)$, hydronephrosis $(n=1)$, uterine fibroid degeneration $(n=2)$, degeneration and torsion of a submucosal uterine fibroid $(n=1)$, simple ovarian cysts $(n=1)$, and ovarian torsion $(n=1)$. Twelve of the 29 patients had normal findings on MR examinations and unremarkable follow-up.

CONCLUSION. The intrinsic safety of MRI and its ability to accurately show abdominal and pelvic disease in pregnant patients make it highly useful in the evaluation of these patients.

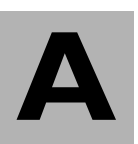
cute abdominal pain in pregnant patients presents a difficult diagnostic challenge. The differential diagnosis during pregnancy is extensive in that the abdominal pain may be obstetric in nature or may be caused by disease of other intraabdominal or intrapelvic structures [1]. Because of the anatomic and physiologic changes that occur with pregnancy, localization of disease can be difficult. The use of conventional radiographic imaging is constrained because of the risk of harm to the fetus by ionizing radiation $[2,3]$.

The use of CT is well established in the evaluation of acute abdominal pain $[4,5]$. CT provides excellent anatomic detail, but a considerable dose of ionizing radiation is con- ferred to the fetus, making this technique undesirable. Sonography is a safe, versatile imaging technique to use in pregnant patients and is often the first imaging technique used in a pregnant patient with abdominal pain. However, in the presence of a gravid uterus, intraabdominal organs may be displaced and challenging to visualize on sonography [6].

MRI provides a good overall topographic display and high intrinsic soft-tissue contrast. MRI also benefits from lack of ionizing radiation $[6,7]$, making it safe to use in pregnant patients. Although a number of prior reports have shown the ability of MRI to evaluate the fetus using current short-duration sequences $[8,9]$, there are fewer reports describing the investigation of maternal abdominal and pel- 


\section{MRI of Pregnant Women with Abdominopelvic Pain}

vic disease on MRI. The purpose of this study was to evaluate the ability of MRI to show causes of acute abdominal and pelvic pain in pregnant patients. To our knowledge, this study is the first in which pregnant patients with acute abdominal or pelvic pain were prospectively evaluated using MRI.

\section{Subjects and Methods \\ Patients}

All abdominal and pelvic MRI studies of pregnant patients who were referred for examination because of acute abdominal or pelvic pain between June 2002 and May 2004 were included $(n=29)$. The term "acute pain" was defined as pain that was sudden in onset and had been present for $72 \mathrm{hr}$ or less. The mean patient age was 25 years (range, 18 35 years), and the mean gestational age was 23 weeks (range $=10-36$ weeks). The use of MRI in these patients was at the discretion of the clinician.

All patients underwent fetal sonographic examination before any other imaging. Six of the 29 patients also underwent complete abdominal sonographic examination before MRI. In the remaining 23 patients, MRI was the choice for primary imaging. Prospective clinical MR interpretations were compared with medical, surgical, and obstetric records when applicable. All patients were followed up until the date of article submission or until the date of the final chart entry (mean follow-up time, 4.1 months; range, 0.5-11 months). The nature of the MRI examination and its associated risks and benefits were explained to all patients before the study, and signed consent was obtained from all patients.

In 22 of the 29 patients, gadolinium was not administered. In the examinations performed with gadolinium $(n=7)$, the risks and benefits of administration of gadolinium were discussed beforehand in detail with the patient and the ordering clinician. The mean gestational age of patients receiving gadolinium was 27 weeks (range, 13-31 weeks). No bowel preparation was used in any study. All MRI studies were performed for clinical indications, so institutional review board (IRB) approval was obtained for case review. A signature waiver was also obtained in accordance with IRB regulations and the Health Insurance Portability and Accountability Act.

\section{MR Technique}

All patients were imaged at the University of North Carolina (UNC) at Chapel Hill or the University of California, San Diego (UCSD). MR examinations of the abdomen and pelvis were performed on a 1.5-T system (VISION or Sonata [UNC] or Symphony [UCSD], Siemens Medical Solutions). All MR examinations were performed using a set protocol including unenhanced T1-weighted im- ages, acquired as a breath-hold spoiled gradientecho sequence (TR range/TE range, 120-170/4.04.5; flip angle, $80-90^{\circ}$ ), and T2-weighted HASTE images (TR/effective TE, infinite/90; 2-3 acquisitions). Section thickness was $7-10 \mathrm{~mm}$, and the matrix size was $128-192 \times 256$ (phase $\times$ frequency encoding) for all sequences.

Immediately after unenhanced images were obtained, an experienced radiologist with fellowship training in body MRI reviewed the images to determine whether a diagnosis could be established without the administration of IV gadolinium chelate (gadodiamide, Omniscan, Amersham Health). In 22 of the 29 MR examinations, gadolinium was not administered. In seven examinations, gadolinium was administered in a dosage of $0.1 \mathrm{mmol} / \mathrm{kg}$ as a rapid bolus injection. Serial spoiled gradientecho images were acquired at $18 \mathrm{sec}$ (late hepatic arterial-arterial-dominant phase) and at 45-60 sec (venous phase). A 90 - to 120 -sec postinjection fatsuppressed spoiled gradient-echo sequence was also acquired. The total examination time was less than 20 min for all studies that did not include IV contrast administration.

\section{Image Interpretation}

All MR examinations were interpreted in a clinical setting at the time of examination by experienced radiologists with fellowship training in body MRI. Radiologists were not blinded to clinical information. Unenhanced images were reviewed first to determine whether a diagnosis could be made without the administration of gadolinium. The same criteria and descriptions of various diseases used in nonpregnant patients [10-17] were used to establish a diagnosis. Medical, surgical, and obstetric records were reviewed to determine the correctness of the clinical MR interpretations. Two radiologists later reviewed the images to obtain measurements and grade signal intensity of visualized disease processes if these determinations were not included in the original clinical interpretations.

\section{Results}

The following disease processes were identified using MRI: appendiceal abscess $(n=1)$ (Fig. 1), appendicitis $(n=2)$, intraabdominal and rectus muscle abscess $(n=1)$, intussusception $(n=1)$ (Fig. 2), pancreatitis $(n=1)$, ulcerative colitis $(n=1)$ (Fig. 3), Crohn's disease with diffuse peritoneal inflammation ( $n$ $=1$ ) (Fig. 4), bilateral adrenal hemorrhage ( $n$ $=1$ ), pyelonephritis $(n=2)$ (Fig. 5), hydronephrosis $(n=1)$, uterine fibroid degeneration $(n=2)$, degeneration and torsion of a subserosal uterine fibroid $(n=1)$, simple ovarian cysts $(n=1)$, and ovarian torsion $(n=1)$ (Fig. 6). Twelve MR examinations were inter- preted as normal. The patients who received IV gadolinium ( $n=7)$ were those with MR diagnoses of appendicitis, appendiceal abscess, ulcerative colitis, Crohn's disease, pyelonephritis (both patients), and hydronephrosis.

The prospective clinical MR interpretations and follow-up medical records were congruent in 28 of 29 patients. The MR interpretations accurately described the type of disease in all but one patient. That particular patient was at 18 weeks' gestation and was referred for MR examination because of acute right lower quadrant pain. Multiple right ovarian cysts were identified on MRI. One month after the MR examination, laparoscopy revealed a torsed right ovary with multiple cysts. The finding of torsion on MRI was neither described prospectively nor seen in retrospect.

MR diagnoses of appendiceal abscess, appendicitis, rectus muscle abscess, intussusception, ovarian torsion, ovarian cyst, uterine fibroid degeneration, and torsed uterine fibroid were confirmed by surgical and pathologic findings. MR diagnoses of Crohn's disease and ulcerative colitis were confirmed by colonic biopsy. Patients with diagnoses of adrenal hemorrhage, pancreatitis, pyelonephritis, and hydronephrosis were confirmed by clinical or laboratory findings (or both). MR diagnosis of ovarian cyst was confirmed with additional imaging postpartum. All patients with MR examinations interpreted as showing normal findings had unremarkable clinical courses according to the follow-up records. No patient with normal findings on MRI was found to have subsequent abdominal or pelvic disease.

The clinical courses for those patients who underwent complete abdominal sonographic examination before MRI are described later; those patients are the ones with remarkable clinical events.

In one patient with pelvic pain at 32 weeks' gestation, MRI showed degeneration and suspected torsion of a subserosal uterine fibroid. Sonography revealed a parauterine mass without Doppler flow but did not show the origin of the mass. MRI showed a clear connection of the mass to the uterus by a narrow pedicle and normal ovaries. Laparotomy revealed a torsed subserosal fibroid rotated $360^{\circ}$ on a very narrow stalk. The patient and infant are well to date.

In one patient with epigastric pain at 35 weeks' gestation, sonographic examination revealed sludge in the gallbladder, mild common bile duct dilatation, and partial visualization of a normal-appearing pancreas. The MR 


\section{Birchard et al.}

examination revealed an abnormal pancreas and surrounding free fluid consistent with pancreatitis. Laboratory values supported the diagnosis of pancreatitis. The patient developed preterm labor the same day and delivered a premature but healthy infant.

In three patients with suspected appendicitis, sonographic examination did not depict the appendix. In the first patient, subsequent MR examination showed a normal appendix. In the second patient, MR examination did not reveal the appendix but did show a lack of pericecal fat stranding. In the third patient, MRI revealed an acutely inflamed, fluidfilled appendix with periappendiceal fat stranding. An open appendectomy was per- formed, and pathology findings confirmed acute appendicitis. All three patients had uneventful deliveries of healthy infants.

In one patient with abdominal and flank pain at 31 weeks' gestation, sonographic examination showed hydronephrosis. The patient underwent MRI to exclude renal abscess. MRI showed hydronephrosis without evidence of pyelonephritis. On the basis of these findings, the patient was treated supportively.

One patient at 31 weeks' gestation was found to have an appendiceal abscess on MR examination. The patient underwent an open appendectomy the next day. The operative notes reported frank pus, a large abscess cavity, and a perforated appendix. At 39 weeks' gestation, the pa- tient underwent uneventful elective repeat cesarean delivery of a healthy infant.

In one patient with diffuse abdominal pain at 13 weeks' gestation, MRI showed findings consistent with ulcerative colitis. The patient was treated with oral steroids. The patient delivered at 35 weeks because of the premature rupture of membranes. Later, colonic biopsy confirmed ulcerative colitis. The infant is healthy to date.

One patient at 31 weeks' gestation was referred for MR examination because of severe upper abdominal pain. MR images revealed bilateral adrenal hemorrhage. The patient was treated with replacement corticosteroids. The patient underwent term elective cesarean de-

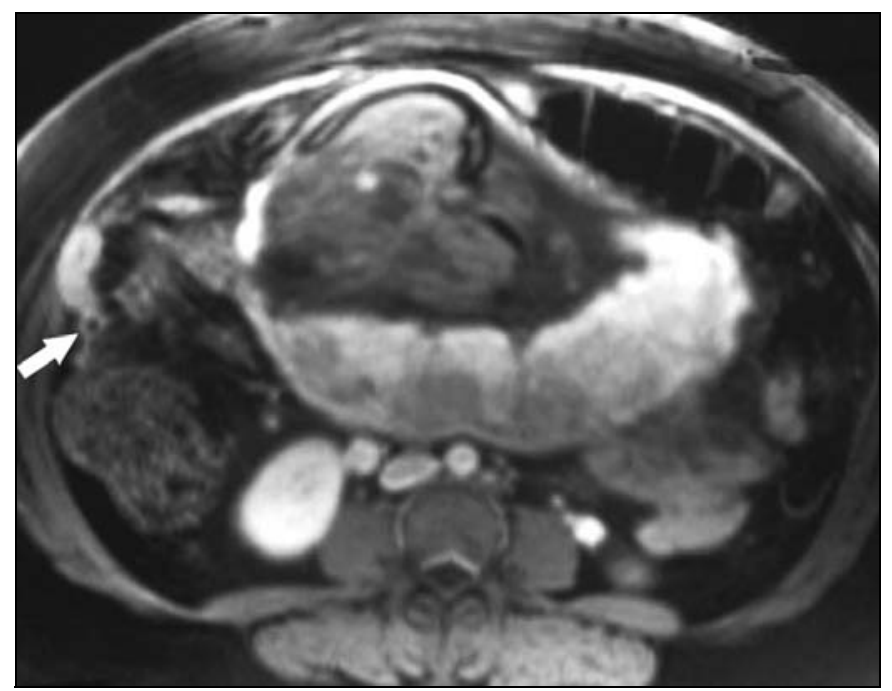

A
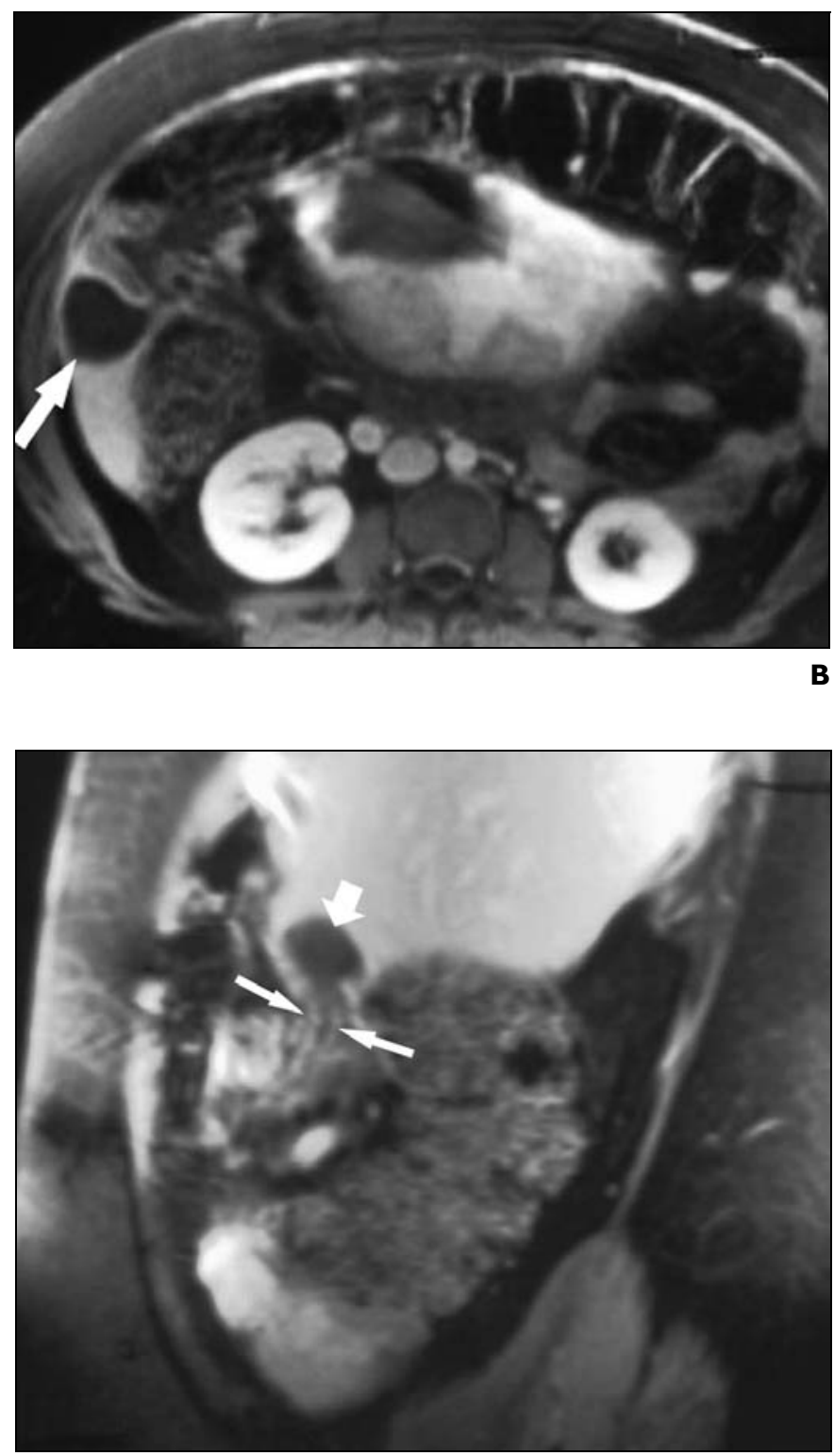

Fig. 1.-27-year-old woman at 31 weeks' gestation with right-sided abdominal pain. A and B, Transverse fat-suppressed images obtained after gadolinium administration show intensely enhancing small segment of intestinal tract (arrow, $\mathbf{A})$ that terminates in subhepatic fluid collection more superiorly (arrow, B).

C. Sagittal fat-suppressed image obtained after gadolinium administration shows continuity between small-caliber viscus structure (thin arrows) and subhepatic fluid collection (thick arrow). 


\section{MRI of Pregnant Women with Abdominopelvic Pain}
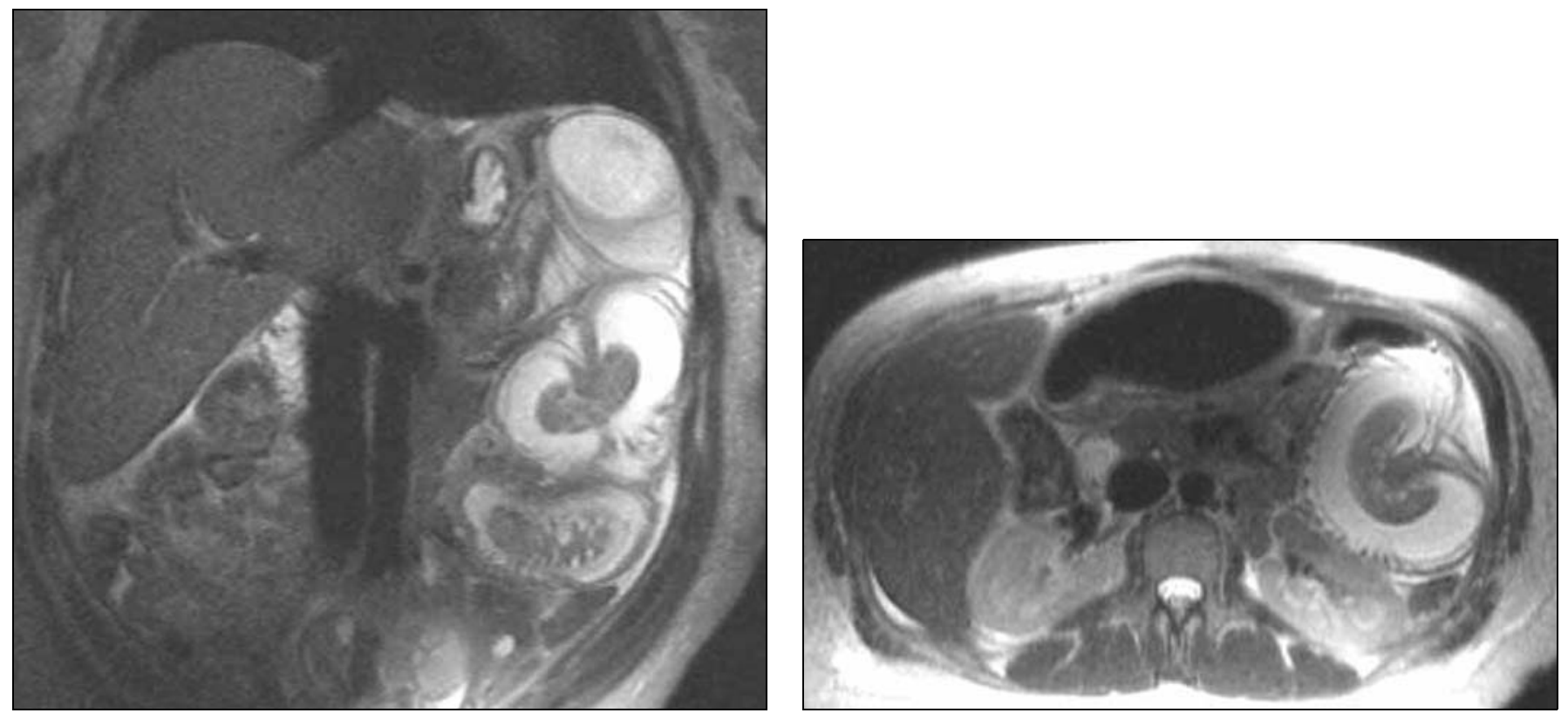

A

Fig. 2. - 31-year-old woman at 23 weeks' gestation with severe left-sided abdominal pain.

A and B, Sagittal and transverse HASTE images show intussusception of small bowel in left upper quadrant. Note dilated loops of small bowel and free fluid.

livery of a healthy infant. Recent clinic notes indicate continued corticosteroid treatment and a healthy infant.

In one patient at 15 weeks' gestation and right lower quadrant pain, MR images showed an intraabdominal and rectus muscle abscess. An abdominal drain was placed into the abscess and remained until labor developed at 36 weeks. The patient underwent cesarean delivery of a premature but healthy infant. A laparoscopic enterolysis performed 3 weeks later revealed a large cavity adherent to the abdominal wall in the right lower quadrant, pericecal inflammation, and a small perforation in the ileum. The patient and infant are well to date.

\section{Discussion}

Although there is substantial literature supporting the ability of MRI to evaluate abdominal and pelvic disease [10-16], there are fewer reports that describe its use in pregnant patients [17-19]. Results of this current study show the ability of MRI to accurately characterize abdominal and pelvic disease in pregnant patients with acute abdominal or pelvic pain. MRI has been shown to be a useful imaging technique for imaging the adrenal gland $[10,11]$ and in the diagnosis of appendicitis $[12,13]$, inflammatory bowel disease [14, 15], pancreatitis [16], intussusception [18], hydronephrosis and pyelonephritis [20, 21], uterine fibroids [22], and adnexal masses [23]. In our study, we used the same criteria
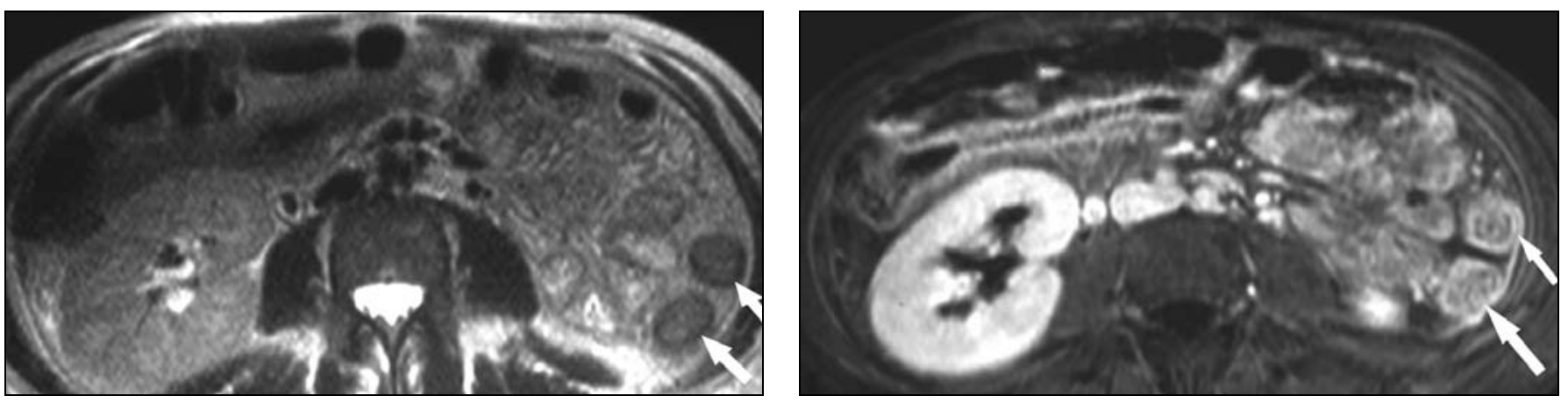

Fig. 3.-29-year-old woman at 14 weeks' gestation with history of abdominal pain and diarrhea.

A, Transverse HASTE image shows bowel wall thickening (arrows).

B, Transverse fat-suppressed image obtained after gadolinium administration shows moderately intense mucosal and serosal enhancement (arrows) of bowel, which is consistent with acute active disease. Submucosal sparing, as shown in this case, is consistent with ulcerative colitis rather than Crohn's disease, which generally shows transmural involvement. 


\section{Birchard et al.}

Fig. 4.-24-year-old woman at 25 weeks' gestation with diffuse abdominal pain and peritoneal signs. Transverse T1-weighted image obtained after gadolinium administration shows free fluid in abdomen, thickening of bowel walls, and diffuse enhancement of peritoneum.

and descriptions of various diseases to establish a diagnosis in pregnant patients.

Acute appendicitis is the most common nonobstetric surgical condition occurring in the obstetric patient population [1, 24]. Anatomic alterations in the location of the appendix accompany an enlarging uterus, usually in the cephalad direction. With appendicitis, a fetal loss rate of less than $2 \%$ is observed with an unruptured appendix, and with a ruptured appendix, the rate is more than $30 \%$ [25]. The diagnosis is commonly missed in pregnant patients because leukocytosis occurs physiologically during pregnancy and may be overlooked; nausea and emesis are common symptoms of appendicitis but are common during pregnancy;

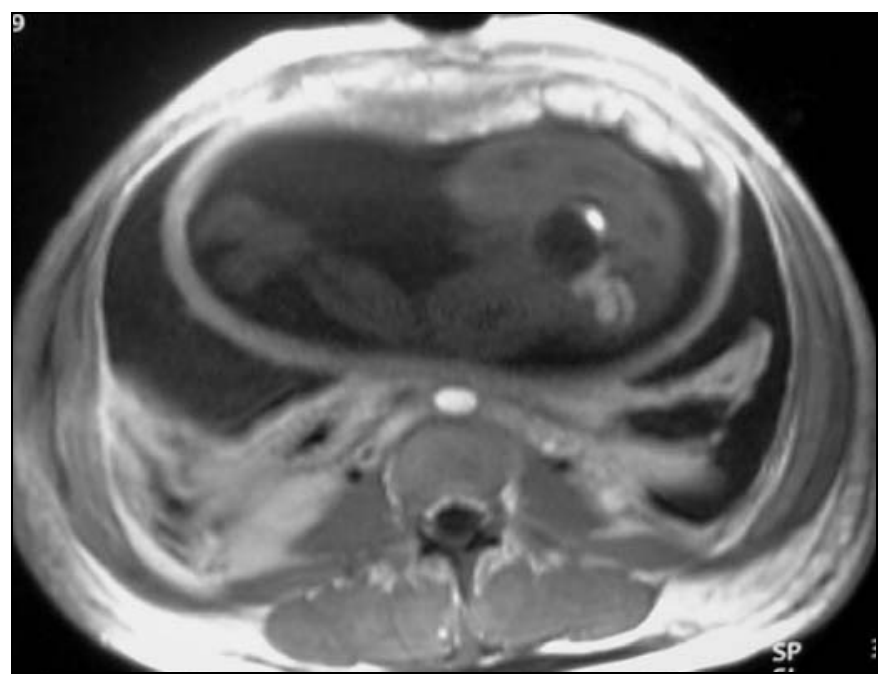

and the location of pain is often not typical [26]. The patient in our study with an appendiceal abscess had cephalad extension of the abscess to a subhepatic location (Fig. 1).

Inflammatory bowel disease, including Crohn's disease and ulcerative colitis, is also a diagnostic challenge during pregnancy [17]. As in appendicitis, many symptoms typical of pregnancy (nausea, emesis, abdominal discomfort) may also be signs of active inflammatory bowel disease. MRI findings such as bowel wall thickening and mucosal enhancement can be seen in inflammatory bowel disease (Figs. 3 and 4). The pharmacologic treatment of inflammatory bowel disease is limited during pregnancy, but in patients with this disease, it is paramount to evaluate for bowel obstruction, abscess, bleeding, and toxic megacolon. Mucosal biopsy is needed to confirm the diagnosis of Crohn's disease or ulcerative colitis.

MRI has been shown to be useful in characterizing uterine fibroids during pregnancy [27] Leiomyomas (fibroids) tend to become smaller during pregnancy, and some involute completely. However, large fibroids can undergo hemorrhagic infarction, which results in pain. Premature labor may ensue as a consequence [28]. Subserosal fibroids can undergo torsion and degeneration during pregnancy. MRI of one patient in our study showed a subserosal uterine mass with signal intensity typical of a degenerating fibroid. Although torsion was suspected
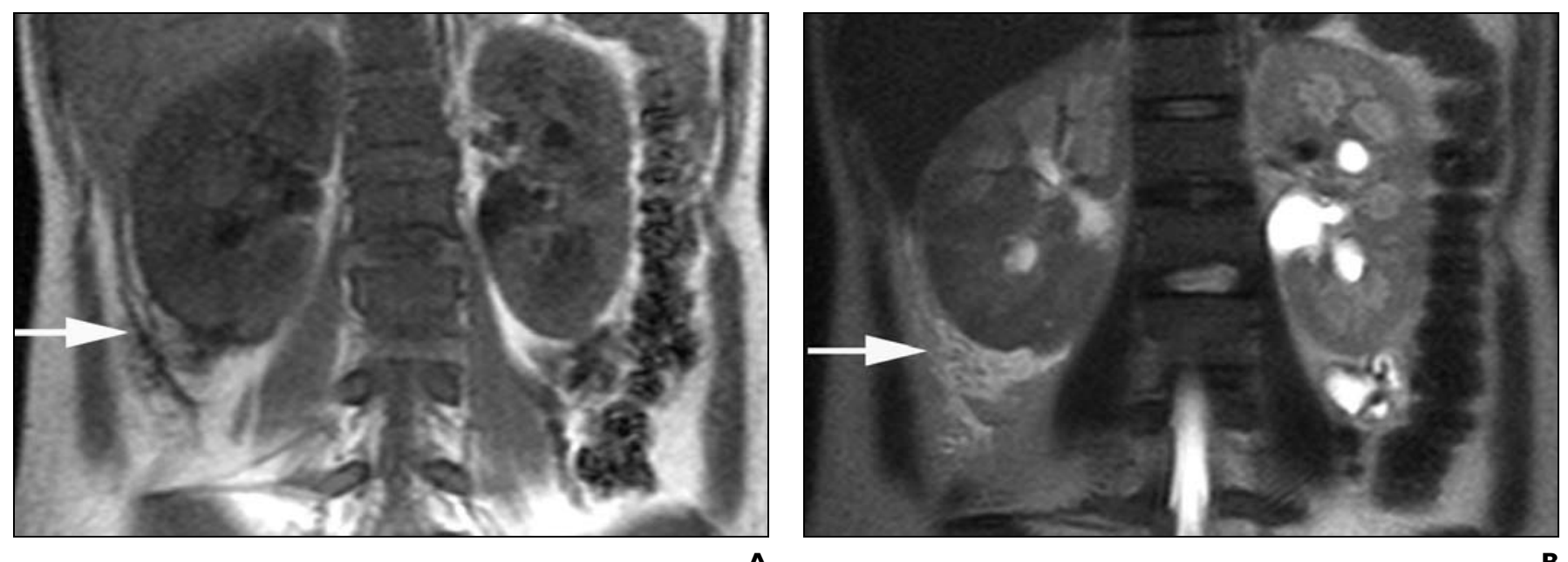

A

Fig. 5.-19-year-old woman at 21 weeks' gestation with right lower quadrant pain and fever.

A and $\mathbf{B}$, Coronal unenhanced T1-weighted (A) and T2-weighted (B) images show enlarged right kidney with surrounding perinephric fluid (arrows), which is consistent with pyelonephritis. 

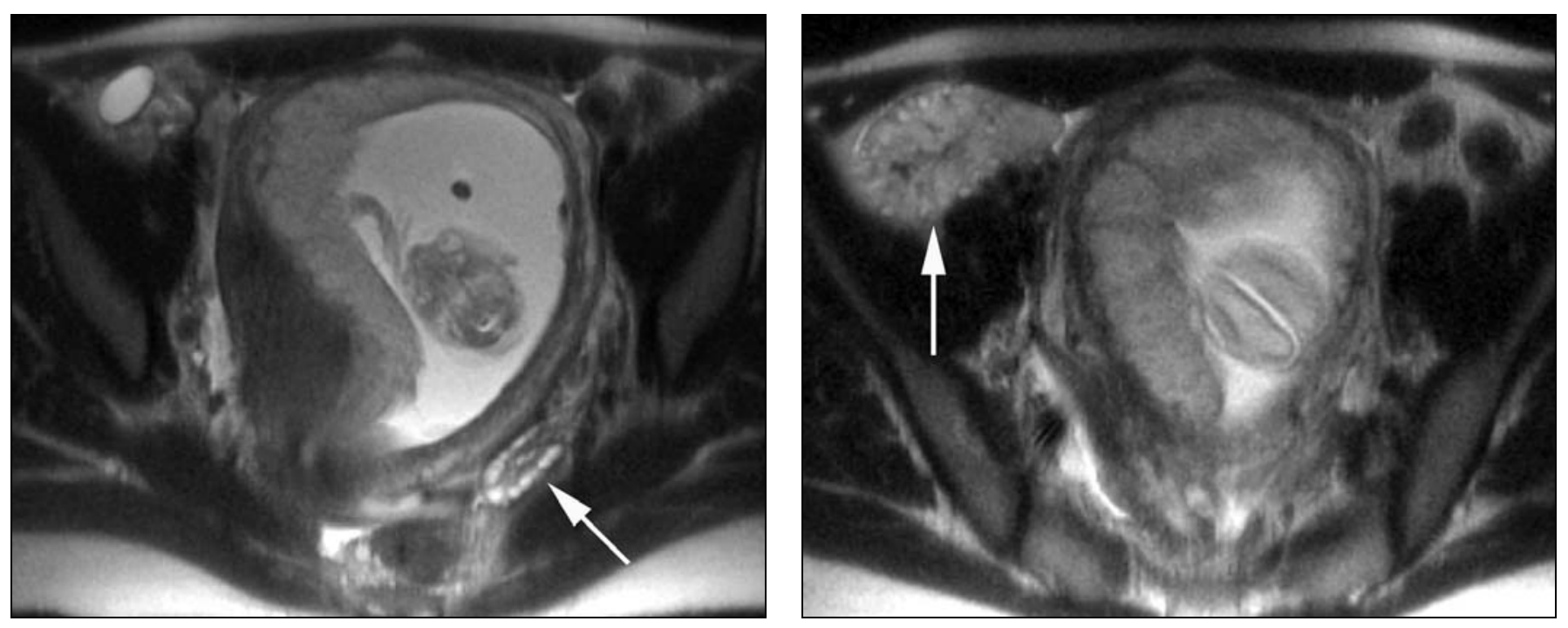

A

Fig. 6. - 30-year-old woman at 15 weeks' gestation with sharp intermittent right lower quadrant pain.

A, Transverse HASTE image shows normal left ovary with normal follicles and stroma (arrow).

B, Transverse HASTE image shows enlarged and edematous right ovary (arrow), which is consistent with torsion.

because Doppler flow was absent on sonography and a narrow stalk was seen on MRI, a more definitive diagnosis of torsion may have been achieved with gadolinium administration. However, discussion with the clinicians after unenhanced imaging indicated that management would not be affected.

Most adnexal masses in pregnancy are asymptomatic [1] and are discovered incidentally during routine sonography examination. The management of painful adnexal masses, however, is controversial. Most adnexal masses are nonneoplastic cysts [1]. Cystic teratomas and cystadenomas are the most common benign ovarian neoplasms during pregnancy [29]. Three percent of ovarian masses during pregnancy are malignant [29]. Torsion must also be considered in pregnant patients because enlargement of the uterus causes a shift in the position of adnexal structures that may then become torsed. The MRI appearance of ovarian torsion is well described [30]. Solid ovarian tissue appears enlarged and edematous. This appearance was observed in one case of ovarian torsion in our series (Fig. 6). In another patient, torsion was not identified, which we believe was due to the fact that the ovary was largely cystic, and therefore edematous tissue was not seen. Torsion of adnexal masses occurs more commonly during pregnancy, and the MRI appearance of torsed and nontorsed structures can be similar. We believe it is important to consider torsion when adnexal masses are discovered in pregnant patients with severe abdominal or pelvic pain.
The most recent available figure for the incidence of acute pancreatitis in pregnant patients is one in 3,333 [31]. Its presentation in pregnancy is similar to that in nonpregnant patients. Gallstones, as in nonpregnant patients, are the most common cause of pancreatitis during pregnancy [32]. Pancreatitis may also be caused by biliary sludge [33], as we believe occurred in the patient in our study. Maternal mortality is low in uncomplicated pancreatitis, but it is greater than $10 \%$ in complicated pancreatitis. The initial diagnosis of pancreatitis is usually clinical, but MRI is useful for characterizing complicated pancreatitis [16].

Adrenal hemorrhage is rare during pregnancy. Most reported cases are associated with preeclampsia or eclampsia, shock, septicemia, and trauma [34]. MR images of the patient in our study showed bilateral adrenal enlargement and signal characteristics consistent with hemorrhage. As in our patient, subsequent adrenal insufficiency may develop, and treatment with replacement corticosteroids is vital.

Hydronephrosis and hydroureter occur in $70-90 \%$ of pregnancies likely because of mechanical obstruction from an enlarged uterus or diminished muscle tone in the urinary tract from hormonal changes associated with pregnancy [35]. In severe cases, patients may develop ureteral obstruction and require ureteral stents or nephrostomy tubes. Acute pyelonephritis occurs in 1-2\% of pregnancies and may result from asymptomatic bacteriuria [35], common in pregnancy. Pyelonephritis (Fig. 5) is associated with premature labor [36] and is important to exclude in pregnant patients.

Gadodiamide (Omniscan, Amersham Health) is a pregnancy category $\mathrm{C}$ drug. It is known to cross the placenta and has been shown to increase skeletal malformations in animal studies (Omniscan, Amersham Health). No controlled longterm studies in pregnant patients have been performed, but it is suggested that gadodiamide be used only during the second and third trimesters of pregnancy and only when the benefits outweigh the risks (Omniscan package insert, Amersham Health). In our study, initial unenhanced images were reviewed in all cases to determine whether a diagnosis could be made without contrast administration. In all cases in which gadolinium was administered, its risks and benefits were discussed in detail with both the patient and the ordering clinician before administration. Longterm animal studies have not been performed to evaluate its carcinogenic potential, but no carcinogenic effects have yet been shown (Omniscan package insert, Amersham Health).

The U.S. Food and Drug Administration guidelines require that MRI devices be labeled to indicate that the safety of MRI with respect to the fetus has not been established [37]. The safety concern arises from the tissue heating effect caused by radiofrequency pulses, which are maximal at the body surface and approach zero near the body core [38]. No adverse fetal effects from MRI have been documented to date. It is generally accepted that MRI should be used judiciously in preg- 


\section{Birchard et al.}

nant patients when benefits outweigh risks as with all imaging techniques [38]. However, we should stress that the single greatest factor in morbidity and mortality of the pregnant patient is delay in diagnosis [39].

One important limitation of our study is the small number of patients. It would be of interest to study a larger population of pregnant patients in the same prospective manner to confirm our initial findings. Furthermore, this study did not include all pregnant patients who presented to our medical centers with acute abdominal or pelvic pain. A blinded prospective study of all these patients would be necessary to further solidify the role of MRI in the evaluation of these patients. Lastly, our study was not performed as a comparison of imaging techniques; instead, we evaluated patients specifically referred for MRI by a clinician. MRI has an established role in the investigation of abdominal and pelvic disease at our centers, and direct referral to MRI by clinicians is not rare. In the future, we believe a comparative study of sonography and MRI would be useful.

In summary, our results show that MRI can be used to identify a wide variety of abdominal and pelvic disease processes in pregnant patients with acute abdominal or pelvic pain. This prospective study shows that abdominal and pelvic diseases in pregnant patients can be well evaluated on MRI. MRI accurately depicted various forms of abdominal and pelvic disease and affected patient management. Correlation with medical records showed correct identification of disease entities in all cases with the exception one, a case of ovarian torsion that was not apparent on MRI.

The pregnant patient with acute abdominal or pelvic pain presents a unique diagnostic challenge. The intrinsic safety and accuracy of MRI in diagnosing abdominal and pelvic disease in pregnant patients make it an excellent choice in the evaluation of these patients.

\section{References}

1. Cappell MS, Friedel D. Abdominal pain during pregnancy. Gastroenterol Clin North Am 2003;32:1-58

2. Wagner LK, Lester RG, Saldana LR. Prenatal risks from ionizing radiations, ultrasound, magnetic fields and radiofrequency waves. In: Wagner LK, Lester RG, Saldana LR, eds. Exposure of the pregnant patient to diagnostic radiations: a guide to medical management, 2nd ed. Madison, WI: Medical Physics Publishing, 1997:77-106

3. Brent RL. The effects of embryonic and fetal ex- posure to x-ray, microwaves, and ultrasound. Clin Perinatol 1986;13:615-648

4. Rosen MP, Sands DZ, Longmaid HE 3rd, Reynolds KF, Wagner M, Raptopoulos V. Impact of abdominal CT on the management of patients presenting to the emergency department with acute abdominal pain. AJR 2000;174:1391-1396

5. Ng CS, Watson CJ, Palmer CR, et al. Evaluation of early abdominopelvic computed tomography in patients with acute abdominal pain of unknown cause: prospective randomised study. $B M J$ 2002;325:1387

6. Kennedy A. Assessment of acute abdominal pain in the pregnant patient. Semin Ultrasound CT MR 2000;21:64-77

7. Dempsey MF, Condon B, Hadley DM. MRI safety review. Semin Ultrasound CT MR 2002;23:392-401

8. Levine D, Barnes PD, Sher S, et al. Fetal fast MR imaging: reproducibility, technical quality, and conspicuity of anatomy. Radiology 1998;206:549-554

9. Kubik-Huch RA, Wisser J, Stallmach T, Ladd ME, Meier A, Marincek D. Prenatal diagnosis of fetal malformations by ultrafast magnetic resonance imaging. Prenat Diagn 1998;11:1205-1208

10. Prokesch RW, Schima W, Berlakovich G, Zacherl J. Adrenal hemorrhage after orthotopic liver transplantation: MR appearance. Eur $J$ Radiol 2001;11:2484-2487

11. Lockhart ME, Smith JK, Kenney PJ. Imaging of adrenal masses. Eur J Radiol 2002;41:95-112

12. Chung JJ, Semelka RC, Martin DR, Marcos HB. Colon diseases: MR evaluation using combined T2weighted single-shot echo train spin-echo and gadolinium-enhanced spoiled gradient-echo sequences. $J$ Magn Reson Imaging 2000;12:297-305

13. Incesu L, Coskun A, Selcuk MB, Akan H, Sozubir S, Bernay F. Acute appendicitis: MR imaging and sonographic correlation. AJR 1997;168:669-674

14. Low RN, Sebrechts CP, Politoske DA, et al. Crohn disease with endoscopic correlation: single-shot fast-spin echo and gadolinium-enhanced fat-suppressed spoiled gradient-echo MR imaging. Radiology 2002;222:652-660

15. Marcos H, Semelka RC. Evaluation of Crohn's disease using half-Fourier RARE gadolinium-enhanced SGE sequences: initial results. Magn Reson Imaging 1999;18:263-268

16. Saifuddin A, Ward J, Ridgway J, Chalmers AG. Comparison of MR and CT scanning in severe acute pancreatitis: initial experiences. Clin Radiol 1993;48:109-116

17. Shoenut JP, Semelka RC, Silverman R, Yaffe CS, Micflikier AB. MRI in the diagnosis of Crohn's disease in two pregnant women. J Clin Gastroenterol 1993; 17:244-247

18. Seidman DS, Heyman Z, Ben-Ari GY, Mashiach $\mathrm{S}$, Barkai G. Use of MRI in pregnancy to diagnose intussusception induced by colonic cancer. Obstet Gynecol 1992;79:822-823

19. Forstner R, Kalbhen CL, Filly RA, Hricak H. Abdominopelvic MR imaging in the nonobstetric evaluation of pregnant patients. AJR 1996;166:1139-1144

20. Katzberg RW, Buonocore MH, Ivanovic M, et al. Functional, dynamic, and anatomic MR urography: feasibility and preliminary findings. Acad
Radiol 2001;8:1083-1099

21. Brown ED, Brown JJ, Kettriz U, Shoenut JP, Semelka RC. Renal abscesses: appearance on gadolinium enhanced magnetic resonance images. Abdom Imaging 1996;21:172-176

22. Murase E, Siegelman ES, Outwater EK, PerezJaffe LA, Tureck RW. Uterine leiomyomas: histopathologic features, MR imaging findings, differential diagnosis, and treatment. RadioGraphics 1999;19:1179-1197

23. Nagayama M, Watanabe Y, Okumura A, Amoh Y, Nakashita S, Dodo Y. Fast MR imaging in obstetrics. RadioGraphics 2002;22:563-580

24. Stone K. Acute abdominal emergencies associated with pregnancy. Clin Obstet Gynecol 2002;45:553-561

25. Mazze RI, Kallen B. Appendectomy during pregnancy: a Swedish registry study of 778 cases. $\mathrm{Ob}$ stet Gynecol 1991;77:835-840

26. Mourad J, Elliot JP, Erickson L, Lisboa L. Appendicitis in pregnancy: new information that contradicts long-held clinical beliefs. Am J Obstet Gynecol 2000;184:954-957

27. Sherer DM, Maitland CY, Levine NF, Eisenberg $\mathrm{C}$, Abulafia O. Prenatal MRI assisting in differentiating between large degenerating intramural leiomyoma and complex adnexal mass during pregnancy. J Matern Fetal Med 2000;9:186-189

28. Coronado GD, Marshall LM, Schwartz SM. Complications in pregnancy, labor, and delivery with uterine leiomyomas: a population-based study. Obstet Gynecol 2000;95:764-769

29. Struyk AP, Treffers PE. Ovarian tumors in pregnancy. Acta Obstet Gynecol Scand 1984;63:421-424

30. Rha SE, Byun JY, Jung SE, et al. CT and MR imaging features of adnexal torsion. RadioGraphics 2002;22:283-294

31. Ramin KD, Ramin SM, Richey SD, Cunningham FG. Acute pancreatitis in pregnancy. Am J Obstet Gynecol 1995;173:187-191

32. Corlett RC Jr, Mishell DR Jr. Pancreatitis in pregnancy. Am J Obstet Gynecol 1972;113:281-290

33. Pazzi P, Gamberini S, Buldrini P, Gullini S. Biliary sludge: the sluggish gallbladder. Dig Liver Dis 2003;35[suppl 3]:S39-S45

34. Cardwell MS. Spontaneous adrenal hemorrhage in pregnancy: a case report. J Reprod Med 1988;33:233-235

35. Puskar D, Balagovic I, Filipovic A, et al. Symptomatic physiologic hydronephrosis in pregnancy: incidence, complications and treatment. Eur Urol 2001;39:260-263

36. Millar LK, Cox SM. Urinary tract infections during pregnancy. Infec Dis Clin North Am 1997;11:13-26

37. U.S. Food and Drug Administration. Magnetic resonance diagnostic device: panel recommendation and report on petitions for MR reclassification. Fed Regist 1998;53:7575-7579

38. Kanal E. Pregnancy and the safety of magnetic resonance imaging. Magn Reson Imaging Clin N Am 1994;2:309-317

39. Devore GR. Acute abdominal pain in the pregnant patient due to pancreatitis, acute appendicitis, cholecystitis or peptic ulcer disease. Clin Perinatol 1980; 7:349-369 